
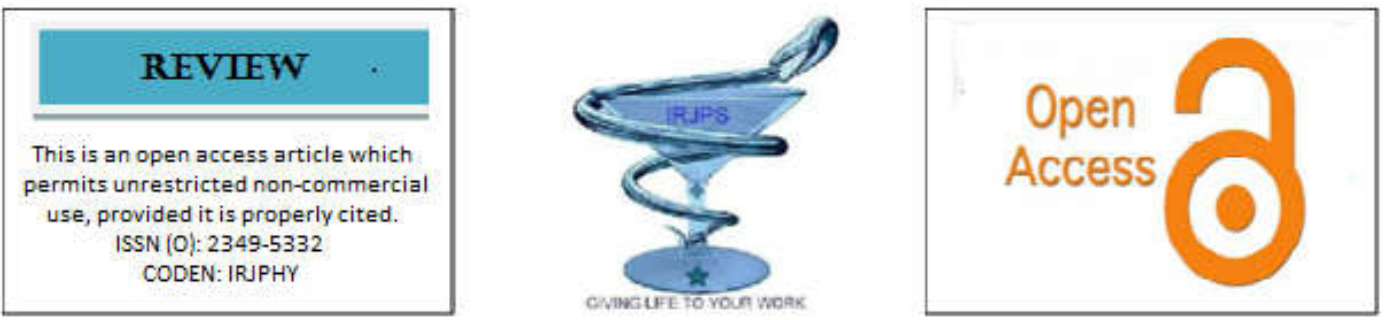

\title{
APPROACHES AND ROLE OF PROTEIN BASED NANOPARTICLES IN DRUG DELIVERY SYSTEM: A REVIEW
}

\author{
Shayeri Chatterjee ${ }^{1 *}$, Beduin Mahanti ${ }^{1}$, Subhabrota Majumdar ${ }^{2}$, Rana Mazumder $^{1}$ \\ ${ }^{1}$ School of Pharmacy, Techno India University, EM 4, Sector-V, Kolkata, West Bengal, India. \\ ${ }^{2}$ Department of Pharmaceutics, Calcutta Institute of Pharmaceutical Technology \& Allied Health Sciences, \\ Banitabla, Uluberia, Howrah, West Bengal, India.
}

Submitted on: 13.06.19;

Revised on: 25.06.19;

Accepted on: 05.07.19

\begin{abstract}
Protein based nanoparticles are investigated as important parts in drug delivery systems, due to their nanoscale size, container-like shape, natural source, biocompatibility, and biodegradability. Nanoparticles which have the range in size from about 1-1000 nanometers in diameter, about one thousand times smaller than the average cell in a human body. These are made up by a variety of materials including metals, polysaccharides, and proteins which having biodegradability, bioavailability, and relatively low cost in nature. protein nanoparticles are easy to process and can be modified by desired specifications such as size, morphology, and weight. Their small size, flexible fabrication, and high surface-area-to-volume ratio make them ideal systems for drug delivery. Nanoparticles can be made from a variety of materials including metals, polysaccharides, and proteins. Biological protein-based nanoparticles such as silk, keratin, collagen, elastin, corn zein, and soy protein-based nanoparticles are advantageous in having biodegradability, bioavailability, and relatively low cost. The key role of protein based nanoparticles has recently use in the nanomedicine era. These nanostructures can be by using protein like albumin, gelatine. The techniques for their fabrication include emulsification, de-solvation, complex coacervation, and electro-spray. The protein-based nanoparticles applications through various routes of administration are explored and reported by researchers which are highlighted in the present review along with the protein nanoparticles as drug delivery carriers.
\end{abstract}

KEYWORDS: Protein based, nanoparticles, nanomedicine, drug delivery system.

Corresponding author: Shayeri Chatterjee

Email: shayeri.ch1992@gmail.com
Indian Research Journal of Pharmacy and Science; 21(2019)1879-1887; Journal Home Page: https://www.irjps.in DOI: $10.21276 /$ irjps.2019.6.2.8 


\section{INTRODUCTION}

In recent years, there has been increased research in drug delivery system by nanoparticles, used as drug carrier system to improve the cellular uptake as well as body distribution. Nanoparticles became the major reason for the change in different properties of many conventional materials which makes them to be more active drug carriers. Several types of nanoparticulate system which includes polymeric nanoparticles, polymeric micelles, solid nanoparticles, lipid-based nanoparticles, for example, solid lipid nanoparticles (SLN), nanostructured lipid carriers (NLC), and lipid drug conjugate (LDC), liposomes, inorganic nanoparticles, dendrimers, magnetic nanoparticles, nanocrystals, and nanotubes.Polymeric nanoparticulate systems from biodegradable and biocompatible polymers are interesting options for controlled drug delivery and drug targeting ${ }^{1,3}$. Polymeric nanoparticles are solid colloidal particles with diameter ranging from 1 to $1000 \mathrm{~nm}$. They have been investigated in drug delivery and drug targeting owing to their particle size and long circulation in the blood $^{4,5}$. They consist of macromolecular materials and can be used therapeutically as adjuvant in vaccines or drug carriers in which the active ingredient is dissolved, entrapped, encapsulated, adsorbed or chemically attached $^{6}$.

Protein polymers are natural macromolecules derived from plants and animals which makes them an easily obtainable, renewable resource. In addition to their biodegradability and tunable properties, nanoparticles fabricated from proteinbased materials are often biocompatible and can be easily processed ${ }^{7,8}$. In this review, the structureand property relationships of these natural proteinbased polymers will be discussed, as well as theirmethods of preparation. The use of these nanoparticles in medicine will then be reviewed with a focuson their application for nanoparticlebased drug delivery.

\section{TYPES OF POLYMERS USED IN PROTEIN BASED NANOPARTICLES}

Various protein polymers vary depending on the application. In this review, silk fibroin ${ }^{9} \operatorname{keratin}^{10}$, collagen $^{11}$, gelatin ${ }^{12}$, elastin $^{13}$, cornzein ${ }^{14}$, and soy $\operatorname{protein}^{15}$ will be given particular attention due to their popularity in biomaterialsresearch. However, additional protein polymers such as $\operatorname{casein}^{16}$, fibrinogen ${ }^{17}$, haemoglobin ${ }^{18}$, bovine serum albumin 19, gluten ${ }^{19}$ have also been used to create nanoparticles.

\subsection{Silk Fibroin}

Silk fibroin protein is among the most popular natural polymers used for the creation ofbiomaterials due to its acceptance by the US Food and Drug Administration (FDA), low cost,and abundance. Commonly extracted from silk produced by the Bombyx mori silkworm, fibroincan be easily isolated after removal of the external sericin protein coating through treatment withsodium carbonate. The resulting fibroin protein is made of semi-crystalline structures comprised ofa light and heavy chain $^{19}$. An isoelectric point (IEP) below pH 7 and molecular weight of $83 \mathrm{kDa}$ have been reported for regenerated silk fibroin, but the latter value may vary depending on theextraction procedure and duration of treatment 20,21 .

\subsection{Keratin}

The use of keratin as a biomaterial has been rapidly expanding because of its abundance, low cost, biocompatibility, and its ability to biodegrade safely ${ }^{22}$. Keratin is a fibrous structural protein with molecular weight of up to $63 \mathrm{kDa}$ and IEP between $\mathrm{pH} 4.5$ and 5 that is derived from the human or animal epidermis and epidermal appendages, such as hair, scales, feathers, and quills in mammals, 
reptiles, and birds ${ }^{23,25}$. The keratin protein is most commonly found in epithelial cells. It is a structural protein that provides the framework for cell-cell adhesion to form a protective layer. Keratin structure is a left-handed alpha-helix which can be coiled together with other keratin proteins to form a polymerized complex and are mainly found in soft tissues. According to recent studies, keratin-based nanoparticles are effective anticancer drug carriers possessing a degree of tumor targeting ability and controlled drug release ${ }^{26}$. The targeting ability of keratin-based nanoparticles is attributed to their $\mathrm{pH}$ sensitivity. Keratin-based nanoparticles can respond to changes in $\mathrm{pH}$ to release their drug contents accordingly in a controlled release. Due to its intrinsic water stability, keratin is also a desirable support polymer for synthetic nanoparticle composites ${ }^{26}$.

\subsection{Collagen and Gelatin}

Collagen is the most abundant biopolymer in the human body ${ }^{27}$. This fibrous protein is a major component of the extracellular matrix and is responsible for maintaining its structure. The majority ofcollagen is located in connective tissues such as the skin, tendons, and ligaments ${ }^{28}$. Collagen can bedivided into two different groups: nonfibrillar and fibrillar, which can be further divided dependingon the structure and use. Due to collagen's biocompatibility and low antigenicity, collagen-based nanoparticles have beenused for the delivery of pharmaceuticals such as theophylline, retinol, tretinoin, and lidocaine.Collagen is capable of resembling the micro environment of some tumors allowing collagen nanoparticles to effectively infiltrate the areas and deliver anticancer therapeutics. Physical properties of collagennanoparticles such as size, surface area, and absorption capacity, are easy to configurate. Like collagen, gelatin has received much attention inthe biomedical field due to its biocompatibility and high abundance. Gelatin contains a triple helicalstructure, similar to collagen, made of repeating amino acids: alanine, glycine, and proline.Depending on the production process, gelatin can be classified as type A or type B and consist ofvarying molecular weights. Gelatin nanoparticles are extensively used as successful anticancerdrug carriers and gene delivery vehicles $^{29}$. Gelatin nanoparticles are able to deliver drugsacross the blood brain barrier, which is a semipermeable barrier that is highly studied for drugdelivery systems. Gelatin nanoparticles have also safely and efficiently carried NS2, a recombinant gene from the hepatitis $\mathrm{C}$ virus, without negatively impacting the function of the gene.

\subsection{Elastin}

Elastin is an important protein found in elastic fibers, specifically in the extracellular matrix.It provides support and elasticity to many structures such as the heart, lungs, skin, and blood vessels with high molecular weight. It is insoluble and therefore can retainits shape and insolubility after stretching. These polypeptides are derived from tropo-elastin, the building block of elastin. Elastinbased proteins also have the ability to communicate with cells through naturally occurringcellular receptors such as elastin binding protein (EBP).The polymer functionality of ELP nanoparticles can be controlledby using a recombinant fabrication technique $^{30,31}$.

\subsection{Corn Zein}

Zein is low molecular weight protein $(20 \mathrm{kDa})$, found within the cytoplasm of corn cell endospermand is insoluble in water except in the presence of alcohol, urea, alkali, and anionic detergents ${ }^{32}$.Zein has a helical wheel shapedstructure with nine homologous units arranged in a non-parallel way with hydrogen bonds stabilizingit. Zein is commonly used in 
fibers, adhesives, plastics, ink, chewing gum, and as a preservative coating for some food and pharmaceuticals. Zein nanoparticles are successful drug carriers for encapsulation and controlled release of fat-soluble compounds such as tocopherol, other proteins, vaccines, and vitamins such as D3.Zein nanoparticles can also have their properties improved by combining thenatural polymer with other substances. For example, sodium caseinate was incorporated with zein nanoparticles to improve particle stability in water.

\subsection{Soy}

Soy protein is a globular protein isolated from soybeans, known as soy protein isolate, and isone of the most abundant types of plant proteins. The globular structure is comprised of two majorsubunits, conglycinin and glycinin, which contain all amino acids particularly glutamate, aspartate, and leucine ${ }^{33}$. Soy protein films, scaffolds, and hydrogels have also been applied in tissue engineering for wound healing and transdermaldrug delivery.Soy protein nanoparticles are becoming more popular due to the high abundance and low cost ofthe protein, as well as its biodegradability and low immunogenicity increasing the loading of enzymes.

\subsection{Other Proteins: Casein, Fibrinogen,}

\section{Hemoglobin, Bovine Serum Albumin, Gluten}

Along with the many proteins mentioned above, there are some that will be excluded from thisreview but are worth mentioning. Casein, fibrinogen, hemoglobin, bovine serum albumin, and glutenare are just a few of many. Similar to those previously explained, the use of these proteins depends ontheir properties and the application's demands. Casein is very useful in hydrophilic environmentssince casein is a hydrophilic protein in itself. It is useful for waterbased environments since as amicrosphere they disperse instead of aggregate. As a micro/nanosphere, fibrinogen polymerizeswhen used in conjunction with a serine protease and forms a protein mesh that can be used to coverand treat open wounds or used in vitro for more in depth biomedical applications $^{34}$. Haemoglobinas a micro/nanoparticle can be used as an oxygen deposit to make oxygen releasing biomaterials.Bovine serum albumin can be used to pack prepared protein particles to aid in protein and drugdelivery ${ }^{35}$. Gluten as a microsphere can be used as a drug delivery vehicle that is very effectivecompared to other widely used proteins.

\section{APPROACHES FOR PROTIEN BASED NANOPARTICLES}

\subsection{Emulsification Method}

In this method, an aqueous phase of albumin was prepared with distilled water and organic phaseplant oil such as cotton seed oil ${ }^{36,37}$. Now the oil and water phase were mixed in the container under mechanical homogenizer until an oil-water (o/w) emlusion was perpared. The above emulsion will be added into the preheat oil over $120 \square \mathrm{C}$ drop by drop. Now there will be evaporation of water and irreversible destruction of albumin which lead to formation of nanoparticles. The resulting particles were suspended into cold ice bath.

\subsection{Desolvation Method}

This method was given by Marty and his coworkers in 1978. This method is also called coacervation method. Under this method, a desolvation agent such as natural salt or alcohol was added into the aqueous solution of albumin ${ }^{38,39}$. By adding of desolvation agents, protein starts changing its structure slowly. Now at certain level protein, clumps will be made and finally nanoparticles will be formed due to crosslinking. To separate the particles, the turbidity of the system should be increased. 
3.3Complex Coacervation Method.This method is generally suited for the DNA entrapment. Since proteins are amphoteric in nature, they can be made cationic or anionic by adjusting the $\mathrm{pH}$. In this method, proteins in aqueous solution were taken; then $\mathrm{pH}$ was adjusted due to the particles with positive charge coming upwards. Then, a mixture of DNA and salt solution was prepared and addedinto the above aqueous protein solution. By the interaction of DNA and protein complex, coacervation occurs. Simultaneously, addition of crosslinker such as 1-ethyl-3(3dimethylaminopropyl) carbodiimide (EDC) was done to conjugate with surface of gelatin nanoparticles and DNA was absorbed.

3.4Electrospray Technique.Electrospray is a new technique used in the preparation of protein nanoparticles. This technique is generally used for gliadin and elastin peptide nanoparticles. In this method, high voltage is applied to the protein solution supplied through an emitter which emits aliquid jet stream through a nozzle which helps in forming an aerosolized size liquid consisting of drug and nucleic acid ${ }^{40,41}$.

\section{ROLE OF PROTEIN BASED NANOPARTICLES}

\subsection{Bioimaging}

In non-specific imaging, thesenanoparticles can be used to dye cells. Data demonstrates that phospholipid encapsulated polymernanoparticles are successful in providing quality fluorescent imaging of cancer cells. These cells displayed no symptoms of toxicity. In addition, it is possible to tune the wavelength emitted byaltering the conjugated protein polymer ${ }^{42}$. In addition, protein nanoparticles have a bright future in targeted cellular imaging. These particles have an increased uptake due to the enhanced permeability and retention of advanced tumors. Near IR light can provide excellent imaging quality when paired with a polymer nanoparticle-based probe due to the previously mentioned properties ${ }^{43}$ Applications these particles in the biomedical imaging field are rapidly growing.

\subsection{Drug Delivery Vehicle}

Protein-based nanoparticles have also found new use as drug delivery vehicles. In additionto their biocompatibility and biodegradability, the surface of protein nanoparticles can be easilyfunctionalized due to their defined primary structure, while charged proteins can facilitate drug loading through electrostatic interactions ${ }^{44}$. The use of natural proteins has also been shown to increase cell retention and reduce the effects of toxic by products produced during degradation ${ }^{45}$. One such protein used to create nanoparticles for drug delivery is corn zein. Due to its hydrophobic nature, this protein is especially suited for the prolonged, controlled release of pharmaceuticals. Lai et al. noted this effect when they used the protein to create nanoparticles loaded with the chemotherapeutic agent, 5-Fluorouracil (5-FU). Corn zein nanoparticles have also been used for the controlled release of vitamin $\mathrm{D}_{3}$, therapeutic proteins such as catalase and superoxide dismutase, and anti-diabetic drugs ${ }^{44}$. Other plant-based proteins such as soy protein have also been used to create nanoparticles for the controlled release of nutrients and pharmaceuticals. Due to soy's balanced composition of nonpolar and polar residues, it can act as a versatile carrier by storing drugs with various functional groups.

\subsection{Biomedical Application}

4.3.1 Routes: There are different applications of protein nanoparticles as carriers for the delivery of proteins, drugs, and peptides via different routes of administration which have been discussed further.

4.3.1.1Oral Route:Oral administration is the most preferred route for any kindof drug applications as 
this route shows different advantages like patient convenience and compliance, avoiding contaminations and infections. The physical barrier is attributed mainly to the continuous monolayer of intestinal epithelial cells which highly express intercellular tight junctions. Physicochemical properties of polymeric NPs can be optimized to facilitate transport across intestinal epithelial cells.

4.3.1.2 Nasal Route: Nasal route is commonly used for non-invasive protein and peptide delivery. Recent advancement in biotechnology, inhalation devices, and targeting motifs has considerably raised research interest in protein and peptide delivery via this route.

4.3.1.3 Pulmonary Route: Pulmonary route is one of themost commonly investigated non-invasive routes to improveabsorption of proteins and peptides. This route providesnumerous advantages including enormous absorptive surfacearea $\left(100 \mathrm{~m}^{2}\right)$, high vascularization, thin alveolar epithelialmembrane $\quad(0.1-0.2 \mu \mathrm{m}), \quad$ and low enzymatic activity despitethese advantages; several factors may regulate pulmonaryprotein and peptide absorption.

4.3.1.4 Blood Brain Barrier Route: Protein nanoparticles have the ability to cross blood brain barrier that cannot becrossed by normal drug through IV injection. The proteinnanoparticle bound drugs include loperamide, tubocurarine, and doxorubicin.

4.3.2OcularTherapy: Protein nanoparticles exhibit a considerablylonger half-life in the eye than eye-

\section{REFERENCES}

1.Gref R., Minamitake Y., Peracchia M.T., Trubetskoy V.,Torchilin V., Langer R., Biodegradable long circulating nanospheres, Science, 1994, 263,1600-3. drops. Pilocarpine bound to gelatin nanoparticles substantially prolonged theintraocular pressure reduction in rabbits with experimentalglaucoma as well as the meiosis time in comparison to apilocarpine eye-drop solution.

4.3.3 Non-viral GeneDelivery: Cationized gelatin nanoparticles have shown the potential of being a new effective carrierfor non-viral gene delivery. The major benefit of gelatine nanoparticle is not only the very low cell toxicity, but alsotheir simple production combined with low cost.

\section{CONCLUSION}

The protein-basedpolymer and protein composite materials are becoming more accepted in the nanoparticle drug delivery system. Their properties are ideal for drug delivery systems and show promise inimproving controlled release or targeting delivery mechanisms. Natural protein polymer is relativelycheap, easy to process, and renewable which makes it an attractive material from an economicperspective. This review focused on the properties of protein materials, such as silk fibroin, keratin, elastin and their usage in nanoparticle drug delivery and biomedical applications. The development of new pharmaceuticals and characteristics of protein nanoparticles must also adapt to provide ideal vehicles for drug delivery. As these new studies emerge and the functionality of these protein materials is improved, more opportunities will be available for effective disease treatment in the future.

2. Labhasetwar V., Song C., Levy R.J., Nanoparticles drug delivery systems, Adv. Drug Deliv. Rev. 1997, 24,63-85.

3. Sinha V.R., Bansal K., Kaushik R., Kumria R., Trehan A., Poly-E-caprolactone microspheres and 
nanospheres: An overview,Int. J. Pharm., $2004,278,1-23$

4. Allemann E., Gurny R., Doelker E., Drug loaded nanoparticles-preparation methods and drug targeting tissues, Eur. J. Pharm. Biopharm.,1993,39,173-91.

5. Yih T.C., Al-Fandi M., Engineered nanoparticles as precise drug delivery systems, J. Cell Biochem., 2006, 97,1184-90.

6. Swarbrick J., Boylan J., Bioavailability of drugs and bioequivalence in encyclopedia of pharmaceutical technology,3rd Edn. InformaHealthcare, USA, 1994,1,164-190

7.Mahmoudi M., Lynch I., Ejtehadi M.R., Monopoli M.P., Bombelli F.B., Laurent S.,Proteinnanoparticleinteractions: Opportunities and challenges, Chem. Rev. 2011, 111, 5610-5637. 8. Weber C.,Coester C., Kreuter J., Langer K.,Desolvation process and surface characterisation of proteinnanoparticles, Int. J. Pharm..2000, 194, 91-102.

9. Wongpinyochit T., Uhlmann P., Urquhart, A.J., Seib F.P., PEGylated silk nanoparticles for anticancer drug delivery, Biomacromolecules,2015, $16,3712-3722$.

10. Zhi X., Wang Y., Li P., Yuan J., Shen J., Preparation of keratin/chlorhexidine complex nanoparticles for long-term and dual stimuliresponsive release, RSC. Adv.,2015, 5, 82334 82341.

11. Posadas I., Monteagudo S., CeñaV., Nanoparticles for brain-specific drug and genetic material delivery, imaging and diagnosis, Nanomedicine ,2016, 11, 833-849.

12. Bajpai A., Choubey J., Design of gelatin nanoparticles as swelling controlled delivery system for chloroquine phosphate, J. Mater. Sci. Mater. Med.,2006, 17, 345-358.

13. Herrero-Vanrell, R., Rincon A., Alonso M., Reboto V., Molina-Martinez I., Rodriguez-Cabello
J., Self-assembled particles of an elastin-like polymer as vehicles for controlled drug release, J. Control. Release, 2005, 102, 113-122.

14. Hurtado-López P., Murdan S.,Zein microspheres as drug/antigen carriers: A study of their degradation and erosion, in the presence and absence of enzymes, J. Microencapsul.,2006, 23, $303-314$

15. Teng Z., Luo Y., Wang Q., Nanoparticles synthesized from soy protein: Preparation, characterization, and application for nutraceutical encapsulation, J. Agric. Food Chem., 2012, 60, 2712-2720.

16. Saralidze K., Koole L.H., Knetsch M.L., Polymeric microspheres for medical applications, Materials,2010, 3, 3537-3564.

17. Paciello A., Amalfitano G., Garzian, A., Urciuolo F., Netti P.A., Hemoglobin-Conjugated GelatinMicrosphere as a Smart Oxygen Releasing Biomaterial, Adv. Healthc. Mater, 2016, 5, 2655 2666.

18. Chen X., Lv G., Zhang J., Tang S., Yan Y., Wu Z., Su J., Wei J., Preparation and properties of BSA-loaded microspheres based on multi-(amino acid) copolymer for protein delivery, Int. J. Nanomed.,2014, 9, 1957.

19. Qi Y., Wang H., Wei K., Yang Y., Zheng R.Y., Kim I.S., Zhang K.Q., A review of structure construction of silk fibroin biomaterials from single structures to multi-level structures, Int. J. Mol. Sci.,2017, 18, 237.

20. Jetbumpenkul P., Amornsudthiwat P., KanokpanontS., Damrongsakkul S., Balanced electrostatic blending approach-An alternative to chemical crosslinking of Thai silk fibroin/gelatin scaffold, Int. J. Biol. Macromol.,2012, 50, 7-13.

21.Wei W., Zhang Y., Shao H., Hu X., Determination of molecular weight of silk fibroin by non-gel sieving capillary electrophoresis, J. AOAC Int.,2010, 93, 1143-1147. 
22.Sharma S., Gupta A., Sustainablemanagement of keratinwaste biomass: Applications and future perspectives, Braz. Arch. Biol. Technol.,2016, 59.

23. Rouse J.G., Van Dyke M.E., A review of keratin-based biomaterials for biomedical applications, Materials, 2010, 3, 999-1014.

24. Thomas P., Said J.W., Nash G., Banks-Schlegel

S., Profiles of keratin proteins in basal and squamous cellcarcinomas of the skin. An immune histochemical study, Lab. Investig. J. Tech. Methods Pathol., 1984, 50, 36-41.

25. Anantha padmanabhan K.P., Lips A., Vincent C., Meyer F., Caso S., Johnson A., Subramanyan K.,Vethamuthu M., Rattinger G., Moore D.J., pHinduced alterations in stratum corneum properties, Int. J.Cosmet. Sci.,2003, 25, 103-112.

26. Li Y., Zhi X., Lin J., You X., Yuan J., Preparation and characterization of DOX loaded keratin nanoparticles for $\mathrm{pH} / \mathrm{GSH}$ dual responsive release, Mater. Sci. Eng., C 2017, 73, 189-197.

27. Nidhin M., Vedhanayagam M., Sangeetha S., Kiran M.S., Nazeer S.S., Jayasree R.S., Sreeram K.J., Nair B.U., Fluorescent nanonetworks: A novel bioalley for collagen scaffolds and Tissue Engineering, Sci. Rep.,2014, 4,5968.

28. Liu C.,CzernuszkaJ., Development of biodegradable scaffolds for tissue engineering: A perspective on emerging technology, Mater. Sci. Technol.,2007, 23, 379-391.

29.Zwiorek K., Kloeckner J., Wagner E., Coester

C.,Gelatin nanoparticles as a new and simple gene delivery system, J. Pharm. Pharm. Sci.,2004, 7, 22 28.

30.Wu Y., MacKay J.A., McDaniel J., Chilkoti A., Clark R.L., Fabrication of elastin-like polypeptide nanoparticles for drug delivery by electro spraying, Biomacromolecules, 2008, 10, 19-24.

31. Pina M.J., Alex S.M., Arias F.J., Santos M., Rodriguez-Cabello J.C., Ramesan R.M., Sharma C.P., Elastin -likere combinamers with acquired functionalities for gene-delivery applications, J.

Biomed. Mater. Res., Part A 2015, 103, 31663178.

32. Liu X., Sun Q., Wang H., Zhang L., Wang J.Y., Microspheres of corn protein, zein, for an ivermectin drug delivery system, Biomaterials,2005, 26, 109-115.

33. Hong C.K., Wool R.P., Development of a biobased composite material from soybean oil and keratin fibers, J. Appl. Polym. Sci., 2005, 95, 1524-1538.

34. Rejinold N.S., Muthunarayanan M., Deepa N., Chennazhi K.P., Nair S.V., Jayakumar R., Development of novel fibrinogen nanoparticles by two-step co-acervation method, Int. J. Biol. Macromol,2010, 47, 37-43.

35. Yu Z., Yu M., Zhang Z., Hong G., Xiong Q., Bovine serum albumin nanoparticles as controlled release carrier for local drug delivery to the inner ear, Nanoscale Res. Lett.,2014, 9, 343.

36. M. Jahanshahi and Z. Babaei, "Protein nanoparticle: a unique system as drug delivery vehicles," African Journal of Biotechnology, vol. 7, no. 25, pp. 4926-4934, 2008.

37. F. Kratz, I. Fichtner, U. Beyer et al., "Antitumour activity of acid labile transferrin and albumin doxorubicin conjugates in in vitro and in vivo human tumour xenograft models," EuropeanJournal of Cancer, vol. 33, pp. 170-175, 1997.

38. J. Sawicka, "Microencapsulation of cholecalciferol by coacervation," Die Pharmazie, vol. 45, no. 4, pp. 264-265, 1990.

39. S. Azarmi, Y.Huang, H. Chen et al., Optimization of a two-step desolvation method for preparing gelatin nanoparticles and cell uptake studies in 143B osteosarcoma cancer cells,Journal ofPharmacy \& Pharmaceutical Sciences, vol. 9, no. 1, pp. 124-132,2006. 
40. Babaei Z., Jahanshahi M., Sanati M.H., Fabrication and evaluation of gelatine nanoparticles for delivering of anti-cancer drug,International Journal of NanoScience and Nanotechnology,2008, 4, 23-29.

41. SchwickH.G., Heide K., Immunochemistry and immunology of collagen and gelatin,Bibliotheca Haematologica, 169, 33,111-125.

42. Howes P., Green M., Levitt J., Suhling K., Hughes M., Phospholipid encapsulated semiconducting polymer nanoparticles: Their use in cell imaging and protein attachment, J. Am. Chem. Soc.,2010, 132, 3989-3996.
43. Yoon S.M., Myung S.J., Kim I.W., Do E.J., Ye B.D., Ryu J.H., Park K., Kim K., Kwon I.C., Kim M.J., Application of near-infrared fluorescence imaging using a polymeric nanoparticle-based probe for the diagnosis and therapeutic monitoring of colon cancer, Dig. Dis. Sci.,2011, 56, 30053013.

44. Xu H., Jiang Q., Reddy N., Yang Y., Hollow nanoparticles from zein for potential medical applications, J. Mater. Chem.,2011, 21, $18227-$ 18235.

45. Lai L.F., Guo H.X. Preparation of new 5fluorouracil-loaded zein nanoparticles for liver targeting. Int. J. Pharm. 2011, 404, 317-323. 